\title{
Facile Synthesis of High Molecular Weight Polypeptides via Surface-Initiated Vapor Deposition Polymerization
}

\author{
Chih-Tsung Yang ${ }^{l, \dagger}$, Jen-Chia $W u^{l, \#}$, and Ying-Chih Chang ${ }^{1,2 *}$ \\ ${ }^{1}$ Genomics Research Center, Academia Sinica, 128 Sec. 2, Academic Rd., Taipei, \\ Taiwan \\ ${ }^{2}$ Department of Chemical Engineering, Stanford University, CA 94309 \\ ${ }^{\dagger}$ Current address: Future Industries Institute and ARC Centre of Excellence in \\ Convergent Bio and Nano Science and Technology, University of South Australia, \\ Mawson Lakes Campus, Mawson Lakes, South Australia 5095, Australia \\ ${ }^{*}$ Current address: Cellmax Life, 18F-1 Park Street, Taipei, Taiwan \\ ${ }^{*}$ Corresponding author email: yingchih@gate.sinica.edu.tw
}

\begin{abstract}
Despite huge effort has been devoted to the design of the initiators and reaction conditions, it remains challenging to synthesize high molecular weight polypeptides with conventional solution phase synthesis. In this work, surface-initiated vapor deposition polymerization (SI-VDP) was utilized to graft synthetic polypeptides poly ( $\gamma$-benzyl L-glutamate) (PBLG) from polystyrene (PS) resin beads by ring-opening polymerization of $\mathrm{N}$-carboxyanhydrides (NCAs). It was demonstrated for the first time that high molecular weight bulk PBLG $(>500,000)$ could be readily obtained within one hour via solvent-free synthetic method which paves the way for the synthesis of copolypeptides with high molecular weight.
\end{abstract}

Keywords: surface-initiated vapor deposition polymerization; N-carboxyanhydrides; ring-opening polymerization; poly ( $\gamma$-benzyl L-glutamate); solvent-free polymerization; solid state polypeptide synthesis 


\section{Introduction}

The study of polypeptides prepared by ring-opening polymerizations (ROPs) of $\alpha$-amino acid N-carboxyanhydrides (NCAs) has flourished in the past decades. ${ }^{1-3}$ As an important and promising strategy in synthetic polypeptides, significant advances aim at gaining precise control over NCAs, known as "living polymerizations". Among them, initiators including primary amine, primary amine hydrochloride salts, ${ }^{5,6}$ and $N$-trimethylsilylamines ${ }^{7,8}$ proved efficient in controlling molecular weight while maintaining low polydispersity (PD) in the synthesis of polypeptides based on solution phase. Nickel catalyst mediated approach was employed to graft surface bound polypeptides from polystyrene (PS) resins, while low molecular weight (MW 22,000, PD 1.52) were measured for the split off graft PBLG. ${ }^{9}$ Nickel mediated planar silicon surface has been exploited to synthesize homo and block coploypeptides and $3.6 \mathrm{~nm}$-thick PBLG film was fabricated in DMF solution within $24 \mathrm{~h},{ }^{10}$ wherein it is still very challenging to synthesize high molecular weight (molecular weight $>$ 200,000) polypeptides in an efficient way.

Polypeptides bearing hydroxylalkanoic acid groups such as poly L-glutamic acid (PLGA) are well known as important synthetic biodegradable polymers. High molecular weight PLGA has been extensively employed in drug delivery and other medical applications due to the biocompatibility and good mechanical properties. ${ }^{11,12}$ The most common synthetic strategy to yield PLGA is through the ROPs of $\gamma$-benzyl-L-glutamate NCA followed by the debenzylation step. ${ }^{13}$ To this end, the synthesis of high MW PBLG is crucial to yield PLGA with high MW. Previously, our lab developed the surface-initiated vapor deposition polymerization (SI-VDP) of $\mathrm{N}$-carboxyanhydride (NCA) of $\alpha$-amino acid technique to successfully fabricate polypeptide brush films from solid substrates in high vacuum, solvent-free conditions. We have reported that, by employing SI-VDP approach, a 187 nm-thick PBLG brush 
film can be achieved within $30 \mathrm{~min},{ }^{14}$ which is in the order of $\mathrm{MW} \sim 3.7 \times 10^{5}$ i.e. DP $\sim 1700$, as estimated by FTIR, ellipsometry, AFM, and XPS. Additionally, the SI-VDP is a living polymerization, where the N-terminal groups of PBLG brush remain active for the subsequent SI-VDP of NCA of amino acid monomers, forming block copolypeptide brushes. ${ }^{15}$ After the debenzylation step, the PLGA polymer brushes can be readily obtained. Despite the versatility of SI-VDP approach, it was largely limited to create "smart surfaces" in which their responsiveness to external stimuli including $\mathrm{pH}$, vapor and temperature, and the cultivability of stem cells were demonstrated. ${ }^{14,16-20}$

In this work, we intend to develop a strategy to employ the SI-VDP of NCA amino acids as a convenient approach to create high MW polypeptides. Specifically, high surface area with the cleavable initiator coated PS resin beads are used for the SI-VDP to create high density PBLG brushes in vacuo (Scheme 1); subsequently, the PBLG may be eluted and collected in bulk.

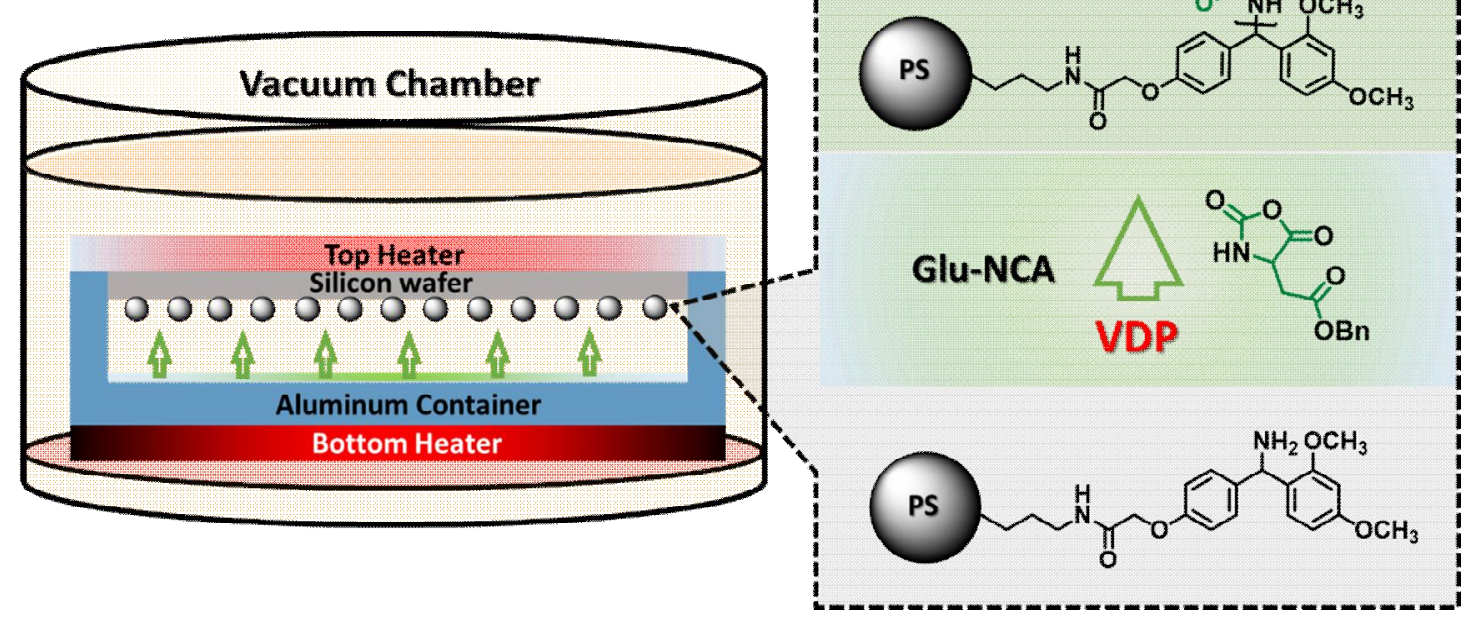

Scheme 1. Experimental setup for synthesis of high molecular weight PBLG on resin beads. 


\section{Experimental Section}

Materials. Commercial solvents and reagents were purchased from Sigma-Aldrich and Acros and used as received. Anhydrous grade solvents were purchased from Acros and were used directly. Rink amide AM resins were purchased from Merck. Undoped, single-polished, wedged silicon (100) wafers (Semiconductor Wafer Inc., with a dimension of $35 \times 15 \times 0.5 \mathrm{~mm}$ ) were used as solid support for resin beads.

Experimental Steps. The NCA of $\gamma$-benzyl L-glutamate (Glu NCA) used for polymerizations from the SI-VDP grafting process were synthesized following the method of Daly and Poché. ${ }^{21}$ The NCA crude product was purified by recrystallization 3 times in a glove box with the mixture of tetrahydrofuran (THF) and hexane. PS resins (100-200 mesh, $0.62 \mathrm{mmol} / \mathrm{g} ; 1 \%$ DVB) was treated with $20 \%$ piperidine/DMF for 30 min and washed with DMF $(3 \times 3 \mathrm{~mL}), \mathrm{CH}_{2} \mathrm{Cl}_{2}(3 \times 3 \mathrm{~mL})$, and then dried in vacuo.

General procedure for vapor deposition polymerization of PBLG. $10 \mathrm{mg}$ of PS resins was swollen in anhydrous THF ( $2 \mathrm{~mL})$ for $30 \mathrm{~min}$, loading on the top of silicon wafer, dried in vacuo for $30 \mathrm{~min} .8 \mathrm{mg}$ of Glu NCA was loaded in the aluminum container and dissolved with $0.3 \mathrm{~mL}$ anhydrous THF. The solution was quickly dried in vacuo, and the wafer was placed on the top of the container with the resin-deposited side facing downward (as shown in Scheme 1). To start the SI-VDP reaction, the chamber was evacuated to $10^{-5}-10^{-4}$ Torr. The temperature of the top heater was raised to 67 ${ }^{\circ} \mathrm{C}$ for $5 \mathrm{~min}$, and then the temperature of the bottom heater was raised to $95{ }^{\circ} \mathrm{C}$ for a controlled time period. After the completion of the reaction, the resin beads were removed from the silicon wafer with DMF. The beads were washed with DMF $(3 \times 5$ $\mathrm{mL})$, THF $(3 \times 5 \mathrm{~mL})$ and then dried in vacuo.

Cleavage. A mixture of TFA/thioanisole/triisopropylsilane/water (85:5:5:5 by vol) 
was added. After $2 \mathrm{~h}$, the resin was washed with TFA $(3 \times 3 \mathrm{~mL})$.

Work-up. The combined solutions were concentrated in vacuo. The residue was washed with ether $(3 \times 3 \mathrm{~mL})$, dried in vacuo and analyzed by GPC and MALDI-TOF/MS.

Characterization. ${ }^{1} \mathrm{H}-\mathrm{NMR}$ was recorded on a Bruker ULTRASHIELD-600 PLUS (600 MHz) spectrometer equipped with a Cryoprobe operating at $298 \mathrm{~K}$. Chemical shifts (in ppm) were assigned according to the internal standard of $\mathrm{CDCl}_{3}(\delta=7.24$ ppm). Attenuated total refractive (ATR) FTIR spectra were recorded with a silicon crystal plate on a Nicolet Magna-IR 860 spectrometer equipped with a MCT detector (32 scans; resolution, $4 \mathrm{~cm}^{-1}$ ). MALDI-TOF mass spectra were measured on Bruker Ultraflex MALDI-TOF mass spectrometer equipped with a nitrogen pulsed laser (355 $\mathrm{nm})$. An equal volume of BSA trypsin digested $(1 \mathrm{pmol} / \mu \mathrm{L})$ solution was mixed homogeneously with $\alpha$-cyano-4-hydroxycinnamic acid (CHCA, $10 \mathrm{mg}$ in acetonitrile/water) solution and was used as the standard for MALDI-TOF mass calibration. Each data point was collected at the average of 500 shots of the laser beam with the laser fluence of $40 \%$. Molecular weights of synthetic polypeptides were measured by gel permeation chromatography (GPC), which was equipped with a solvent delivery system, two columns $(300 \times 7.8 \mathrm{~mm}, 5 \mu \mathrm{m}$, phenomenex $)$, and a differential refractometer (RI). Two columns were maintained at $40{ }^{\circ} \mathrm{C}$. THF was used as the eluent at a flow rate of $1.0 \mathrm{~mL} / \mathrm{min}$. The sample concentration for GPC measurements was $2 \mathrm{mg} / \mathrm{mL}$ THF. Pure styrene and six PS standards with molecular weights ranged from 104 to $382100 \mathrm{~g} / \mathrm{mol}$ were used for calibration.

\section{Results and Discussion}

The synthetic PBLG brushes were successfully synthesized from resin beads by SI-VDP and confirmed by FTIR characterization, as shown in Figure 1. Absorption 
bands for amide I at $1652 \mathrm{~cm}^{-1}$ and amide II at $1547 \mathrm{~cm}^{-1}$ were indicative of the $\alpha$-helical conformation of synthetic PBLG on solid substrates and the distinctive absorption bands of the synthetic PBLG cleaved from resin beads are also consistent with that of the bulk PBLG. ${ }^{22}$ The results of synthetic PBLG by SI-VDP were categorized into two groups according to different variables including resin amounts, reaction time, and temperature (data not shown here): (i) At a fixed temperature, we loaded resin beads with the averaged amount of $10 \mathrm{mg}$ to investigate the effects of time on the synthesis of PBLG (Table 1). The obtained MW and yield are also plotted as a function of reaction time (Figure 2). The MW of synthetic PBLG was significantly increased to $1 \times 10^{6}$ with the prolonged reaction time from 10 min to 60 min, demonstrating the ability to synthesize high MW PBLG in an efficient way. The total yield was increased over 30 min of reaction time. The PDI in all samples are $\sim 3$ to $\sim 5$; it is most likely caused by the physical hindrance and inhomogeneous local monomer vapor concentration, as the resin beads were dispersed randomly on the silicon wafer in our current experimental setup.

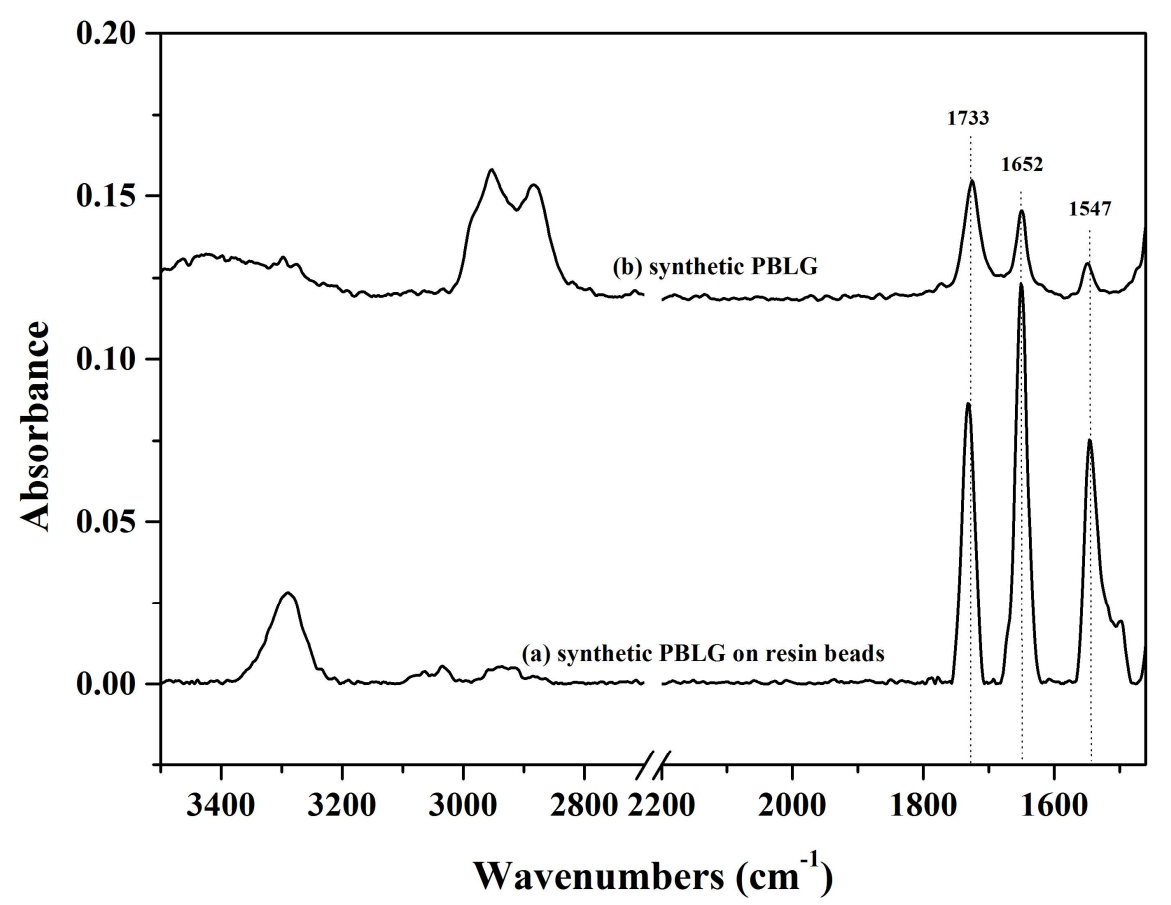

Figure 1. ATR-IR spectra of (a) grafted PBLG from PS resins, (b) synthetic PBLG cleaved from PS 
resins.

Table 1. Effects of reaction time on the synthesis of $\mathrm{PBLG}^{a}$

\begin{tabular}{ccccccc}
\hline Entry & $\begin{array}{c}\text { Time } \\
(\mathrm{min})\end{array}$ & $\begin{array}{c}\text { Resin amount } \\
(\mathrm{mg})\end{array}$ & $\begin{array}{c}\text { Yield } \\
(\mathrm{mg})\end{array}$ & $\mathrm{Mn}$ & $\mathrm{Mw}$ & $\mathrm{PDI}^{b}$ \\
\hline 1 & 10 & 10.2 & 3.4 & 9,040 & 42,100 & 4.657 \\
2 & 10 & 10.3 & 3.6 & 7,740 & 41,900 & 5.413 \\
3 & 30 & 9.6 & 4.3 & 52,000 & 166,600 & 3.204 \\
4 & 30 & 9.8 & 4.5 & 58,000 & 280,100 & 4.829 \\
5 & 60 & 9.0 & 4.1 & 331,900 & $1,217,000$ & 3.667 \\
6 & 60 & 9.6 & 4.5 & 186,000 & 753,100 & 4.049 \\
\hline
\end{tabular}

${ }^{a}$ Reaction condition: $67{ }^{\circ} \mathrm{C}$ (top heater) $/ 95^{\circ} \mathrm{C}$ (bottom heater), vacuum pressure $=5 \times$ $10^{-5}-1 \times 10^{-4}$ Torr. ${ }^{b}$ Calculated by GPC (eluent: THF, $c=2 \mathrm{mg} / \mathrm{mL}$, calibrated with polystyrene standards). 


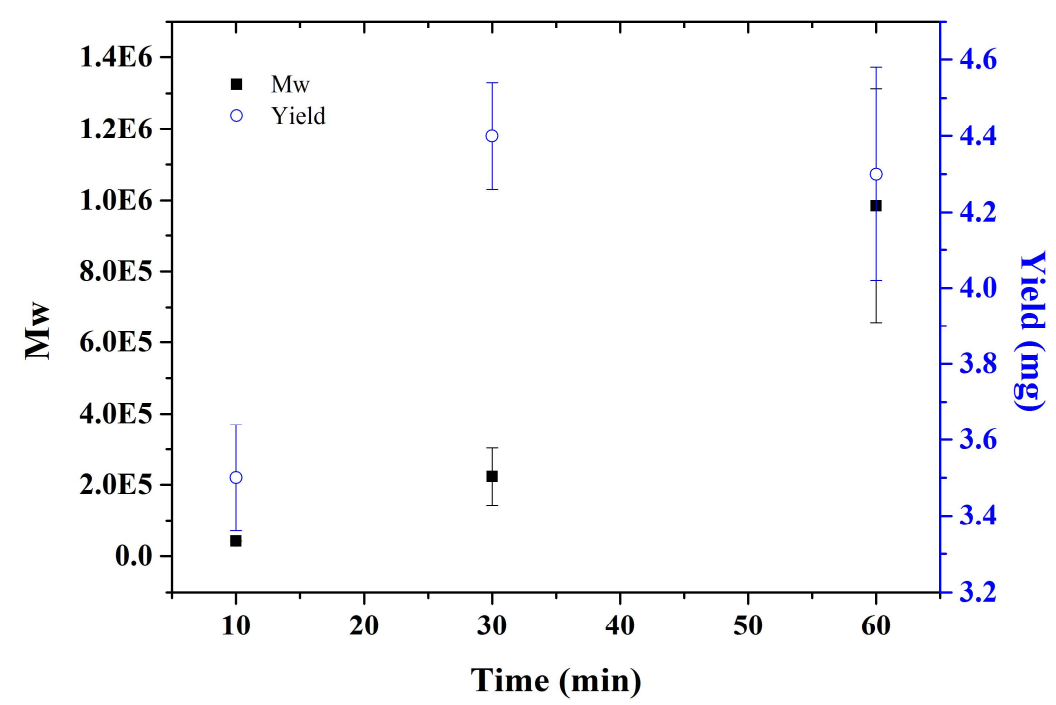

Figure 2. Plot of obtained molecular weight and yield of PBLG as a function of time.

(ii) We further examine the effect of resin amount to the final MW and yields. As shown in Table 2 and Figure 3, at a fixed 60 min reaction time, with the increase of resin amounts from $5.8 \mathrm{mg}$ to $9.6 \mathrm{mg}$, the yields of PBLG were increased by $\sim 150 \%$, and the average Mn were also increased from the order of $10^{5}$ to $\sim 10^{6}$. It is also worth noted that PD distribution of synthetic PBLG resemble that of polycondensates, with typical PD $\geqq 2 \cdot 0 .^{23,24}$

Table 2. Effects of resin amounts on the resulting $\mathrm{PBLG}^{a}$

\begin{tabular}{cccccc}
\hline Entry & $\begin{array}{c}\text { Resin amount } \\
(\mathrm{mg})\end{array}$ & $\begin{array}{c}\text { Yield } \\
(\mathrm{mg})\end{array}$ & $\mathrm{Mn}$ & $\mathrm{Mw}$ & $\mathrm{PD}^{b}$ \\
& 5.8 & 2.8 & 77,500 & 198,100 & 2.556 \\
1 & 5.8 & 2.7 & 191,300 & 359,100 & 1.877 \\
2 & 9.0 & 4.1 & 331,900 & $1,217,000$ & 3.667 \\
3 & 9.6 & 4.5 & 186,000 & 753,100 & 4.049 \\
4 & 14.0 & 4.6 & 188,500 & 778,200 & 4.128 \\
5 & 15.7 & 5.4 & 559,800 & $1,012,000$ & 1.808 \\
\hline
\end{tabular}


${ }^{a}$ Reaction condition: $67{ }^{\circ} \mathrm{C}$ (top heater) $/ 95^{\circ} \mathrm{C}$ (bottom heater), $5 \times 10^{-5} \sim 1 \times 10^{-4}$ Torr. $60 \mathrm{~min}$ reaction time ${ }^{b}$ Calculated by GPC (eluent: THF, c $=2 \mathrm{mg} / \mathrm{mL}$, calibrated with PS standards).

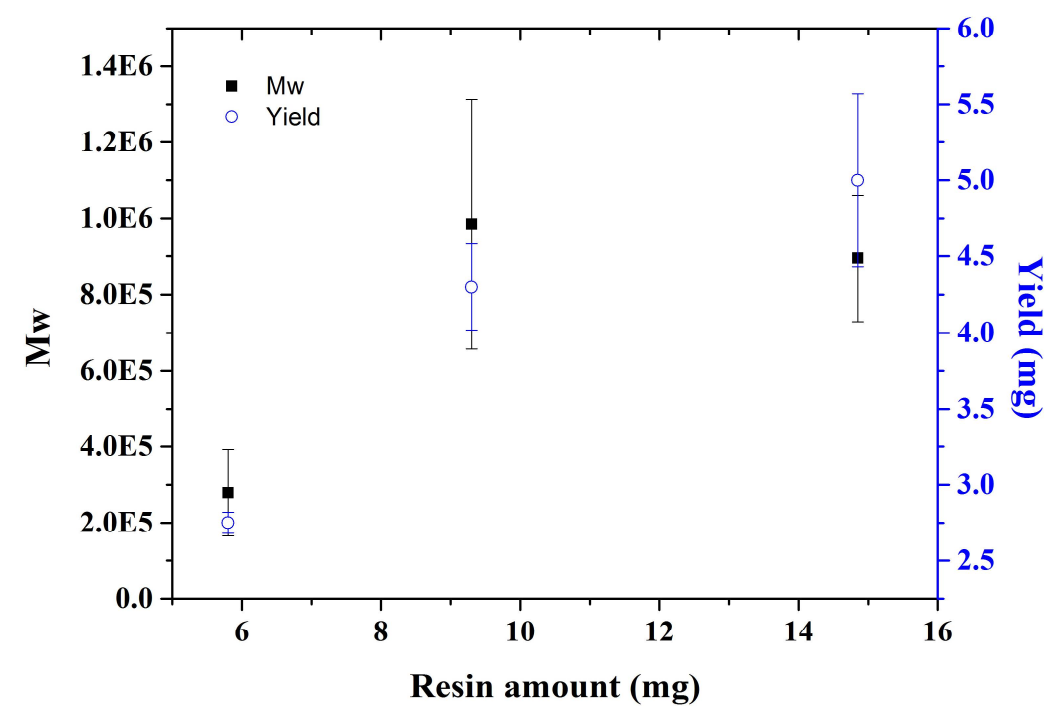

Figure 3. Plot of obtained molecular weight and yield of PBLG as a function of resin amount.

MALDI-TOF mass spectroscopy was employed to gain the mechanistic insight of SI-VDP process (Figure 4). Two periodic values of PBLG were observed as a result of two different chain terminations. In series 1 (denoted as white diamond), the periodic $\mathrm{m} / \mathrm{z}$ values of 2011, 2230, 2449 and 2668, respectively, were observed. These signals are located with $219 \mathrm{Da}$, the molecular weight of the repeat unit of PBLG, and with a primary amine end group. In series 2 (denoted as black diamond), they included the same repeat unit of PBLG but with a lactam termination. This may be because during the heating process of polymerization, PBLG can undergo an intramolecular termination step leading to a 5-membered lactam structure which was formed by intramolecular nucleophilic attack of the terminal amine group to the benzyl ester in the side chain. ${ }^{25}$ The MALDI-TOF mass data shown were in good agreement with the assumption. The major products of the synthetic PBLG has a primary amine chain end and even the lactam structure can be hydrolyzed by alkaline to generate primary amine which is capable of grafting the block copolypeptides on resin beads in the 
future. Additionally, considering the efficiency in producing PBLG of MW > 300,000 (DP 1400), the VDP system is 72 times faster than the amine initiators used previously in solution phase. ${ }^{13}$

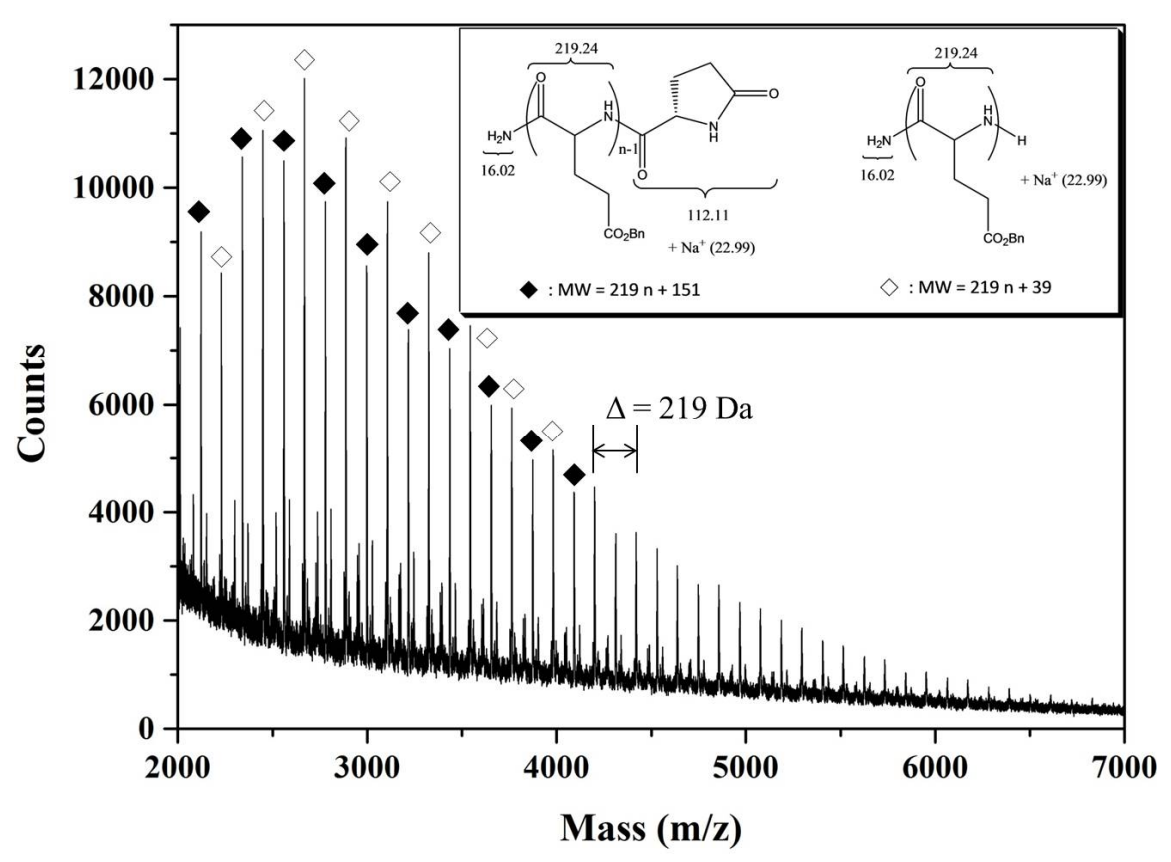

Figure 4. MALDI-TOF mass spectrum of synthetic PBLG prepared by VDP.

Finally, as compared with our previous study on the synthesis of PBLG grafting from planar substrates, shown in Figure 5, we used "solvent quench" approach to promote molecular bundles of PBLG with the average tilt angle $\left\langle\theta>\right.$ of $30^{\circ}$ stretching from silicon surface normal. ${ }^{26,27}$ The film thickness visualized by ellipsometry was approximate $300 \mathrm{~nm}\left(\mathrm{DP} \sim 2300\right.$, i.e. $\left.\mathrm{MW} \sim 5.0 \times 10^{5}\right)$. The MW range of synthetic bulk PBLG is consistent with that on silicon wafer, suggesting that VDP system could be utilized in grafting high MW PBLG using various solid substrates. 


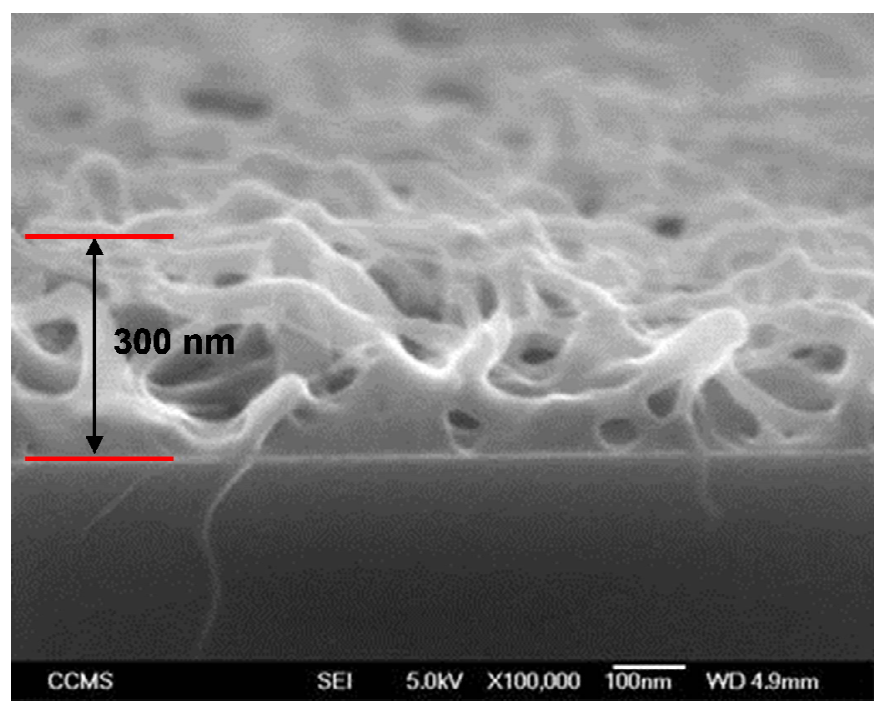

Figure 5. SEM image of "quenched" PBLG grafted on silicon wafer.

\section{Conclusions}

In this paper, a new synthetic method that employs NCAs developed in order to overcome challenges in the synthesis of high MW bulk polypeptides that conventional strategies fail to achieve. It demonstrated the rapid polymerization to yield PBLG in vacuo, completing within 1h. Precise controlled PDI and the synthesis of copolypeptides are the future studies using our optimized system.

\section{Acknowledgement}

We thank Ministry of Science and Technology, Taiwan (MOST104-2113-M -001-015-MY3) and Academia Sinica for financial support. Dr. Mo-Yuan Shen (Stanford) for the comments and suggestions. 


\section{References}

(1) Kricheldorf, H. R. Polypeptides and 100 years of chemistry of alpha-amino acid N-carboxyanhydrides Angew. Chem. Int. Edit. 2006, 45, 5752-5784.

(2) Deng, C.; Wu, J.; Cheng, R.; Meng, F.; Klok, H.-A.; Zhong, Z. Functional polypeptide and hybrid materials: Precision synthesis via $\alpha$-amino acid N-carboxyanhydride polymerization and emerging biomedical applications Prog. Polym. Sci. 2014, 39, 330-364.

(3) Wibowo, S. H.; Sulistio, A.; Wong, E. H.; Blencowe, A.; Qiao, G. G. Polypeptide films via N-carboxyanhydride ring-opening polymerization (NCA-ROP): past, present and future Chem. Comm. 2014, 50, 4971-4988.

(4) Zhao, W.; Gnanou, Y.; Hadjichristidis, N. Fast and Living Ring-Opening Polymerization of $\alpha$-Amino Acid N-Carboxyanhydrides Triggered by an "Alliance" of Primary and Secondary Amines at Room Temperature Biomacromolecules 2015, 16, 1352-1357.

(5) Dimitrov, I.; Schlaad, H. Synthesis of nearly monodisperse polystyrene-polypeptide block copolymers via polymerisation of N-carboxyanhydrides Chem. Comm. 2003, 2944-2945.

(6) Dimitrov, I.; Kukula, H.; Colfen, H.; Schlaad, H. Advances in the synthesis and characterization of polypeptide-based hybrid block copolymers Macromol. Sy. 2004, 215, 383-393.

(7) Lu, H.; Cheng, J. J. Hexamethyldisilazane-mediated controlled polymerization of alpha-Amino acid N-carboxyanhydrides J. Am. Chem. Soc. 2007, 129, 14114-+.

(8) Lu, H.; Cheng, J. J. N-trimethylsilyl amines for controlled ring-opening polymerization of amino acid $\mathrm{N}$-carboxyanhydrides and facile end group functionalization of polypeptides J. Am. Chem. Soc. 2008, 130, 12562-+.

(9) Witte, P.; Menzel, H. Nickel-mediated surface grafting from polymerization of alpha-amino acid-N-carboxyanhydrides Macromol. Chem. Phys. 2004, 205, 1735-1743.

(10) Sparks, B. J.; Ray, J. G.; Savin, D. A.; Stafford, C. M.; Patton, D. L. Synthesis of thiol-clickable and block copolypeptide brushes via nickel-mediated surface initiated polymerization of alpha-amino acid N-carboxyanhydrides (NCAs) Chem. Comm. 2011, 47, 6245-6247.

(11) Duro-Castano, A.; Conejos-Sánchez, I.; Vicent, M. J. Peptide-based polymer therapeutics Polymers 2014, 6, 515-551.

(12) Baumgartner, R.; Kuai, D.; Cheng, J. Synthesis of controlled, 
high-molecular weight poly (L-glutamic acid) brush polymers Biomater. Sci. 2017, 5, 1836-1844.

(13) Li, H.; Niu, Y. Synthesis and Characterization of Poly (L-glutamic acid) with High Molecular Weight Polym. Plast. Technol. Eng. 2012, 51, 1062-1067.

(14) Wang, Y. L.; Chang, Y. C. Grafting of homo- and block co-polypeptides on solid substrates by an improved surface-initiated vapor deposition polymerization Langmuir 2002, 18, 9859-9866.

(15) Wang, Y.; Chang, Y. C. Patterning of Polypeptide Thin Films by the Combination of Surface-Initiated Vapor-Deposition Polymerization and Photolithography Adv. Mater. 2003, 15, 290-293.

(16) Chang, Y. C.; Frank, C. W. Vapor deposition-polymerization of alpha-amino acid N-carboxy anhydride on the silicon(100) native oxide surface Langmuir 1998, 14, 326-334.

(17) Wang, Y. L.; Chang, Y. C. Synthesis and conformational transition of surface-tethered polypeptide: Poly(L-glutamic acid) Macromolecules 2003, 36, 6503-6510.

(18) Yang, C. T.; Wang, Y. L.; Yu, S.; Chang, Y. C. I. Controlled Molecular Organization of Surface Macromolecular Assemblies Based on Stimuli-Responsive Polypeptide Brushes Biomacromolecules 2009, 10, 58-65.

(19) Yang, C. T.; Wang, Y. L.; Chang, Y. C. Effect of Solvents and Temperature on the Conformation of Poly(beta-benzyl-L-aspartate) Brushes Biomacromolecules 2010, 11, 1308-1313.

(20) Yang, C.-T.; Wang, Y.; Frank, C. W.; Chang, Y.-C. Chemoresponsive surface-tethered polypeptide brushes based on switchable secondary conformations RSC Adv. 2015, 5, 86113-86119.

(21) Daly, W. H.; Poche, D. The preparation of N-carboxyanhydrides of alpha-amino-acids using bis(trichloromethyl)carbonate Tetrahedron Letters 1988, 29, 5859-5862.

(22) Chang, Y.-C.; Frank, C. W. Grafting of poly ( $\gamma$-benzyl-l-glutamate) on chemically modified silicon oxide surfaces Langmuir 1996, 12, 5824-5829.

(23) Flory, P. J. Fundamental principals of condensation polymerization Chem. Rev. 1946, 39, 137-197.

(24) Kricheldorf, H. R.; Schwarz, G. Cyclic polymers by kinetically controlled step-growth polymerization Macromol. Rapid Comm. 2003, 24, 359-381.

(25) Kamei, Y.; Sudo, A.; Endo, T. Synthesis of Polypeptide Having Defined Terminal Structures Through Polymerization of Activated Urethane-Derivative of gamma-Benzyl-L-glutamate Macromolecules 2008, 41, 7913-7919.

(26) Wang, Y. L.; Chang, Y. C. Preparation of unidirectional end-grafted 
alpha-helical polypeptides by solvent quenching J. Am. Chem. Soc. 2003, 125, 6376-6377.

(27) Wu, J. C.; Chen, C. C.; Chen, K. H.; Chang, Y. C. Controlled growth of aligned alpha-helical-polypeptide brushes for tunable electrical conductivity Appl. Phys. Lett. 2011, 98. 


\section{Table of Contents}

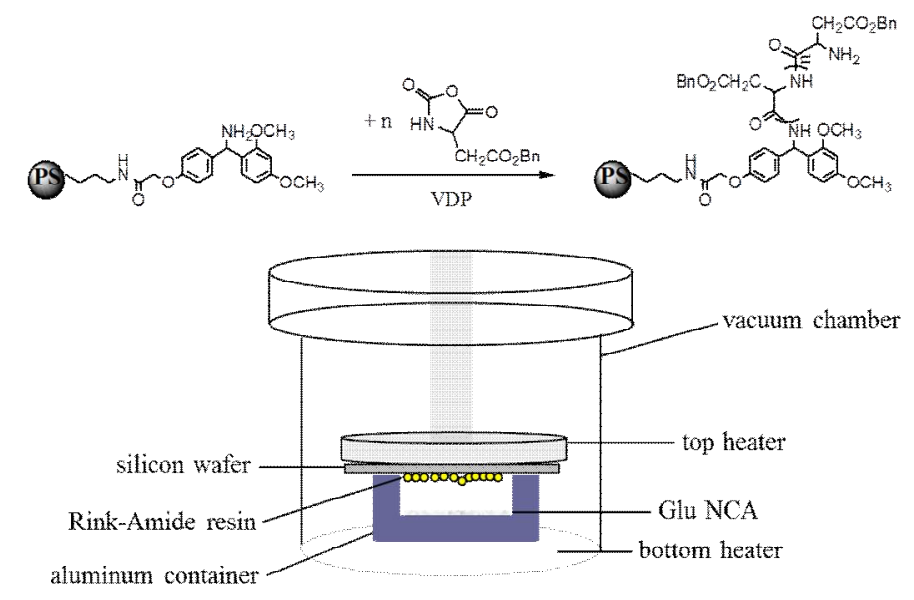

